\title{
Molecular characterization of Eimeria spp. from chicken by Polymerase Chain Reaction based on species-specific SCAR markers
}

\author{
AMAM Zonaed Siddiki ${ }^{1 *}$, SohanaAkter Mina ${ }^{2}$, Md. Anayet Hasan ${ }^{2}$, Mohammed \\ Touaha Akbar ${ }^{2}$, Rashel Alam ${ }^{2}$, Md. Ashraful Islam², Israt Zahan Ira ${ }^{3}$, Bibi Ayesa ${ }^{2}$ \\ ${ }^{I}$ Department of Pathology and Parasitology, Chittagong Veterinary and Animal Sciences University, \\ Chittagong-4225, Bangladesh \\ ${ }^{2}$ Department of Genetic Engineering and Biotechnology, Faculty of Biological Sciences, University of \\ Chittagong, Chittagong-4331, Bangladesh \\ ${ }^{3}$ Department of Clinical Pharmacy and Pharmacology, Noakhali Science and Technology University, Noakhali, \\ Bangladesh
}

\begin{abstract}
Coccidiosis in chickens is one of the major problems of poultry industry that is caused by protozoan parasites of genus Eimeria. Present study was conducted to characterize the Eimeria species infecting poultry in different regions of Chittagong district of Bangladesh. Seven species of Eimeria including E. tenella, E. necatrix, E. acervulina, E. maxima, E. brunetti, E. mitis, and E. praecox have been characterized using conventional PCR analysis through amplifying unique single copy sequences derived from sequence characterized amplified region (SCAR) markers. The present study is the first of its type to use molecular tools to identify Eimeria spp. infecting chicken in Bangladesh. The study indicates that the modern molecular technique involving PCR to detect the Eimeria species are more reliable and accurate than the traditional morphology-based technique.
\end{abstract}

Keywords:Eimeria , PCR, SCAR, Coccidiosis, Bangladesh

\section{Introduction}

Coccidiosis is recognized as the parasitic disease and is caused by the Apicomplexan parasites, Eimeria species. Coccidiosis has a greatest economic impact on poultry industry and is one of the most common infectious disease found in chickens [1]. It causes great loss in the poultry industry due to morbidity, mortality, poor growth, disability in digestion and reduction in egg production [2]. The life cycle of these parasites is complex, which ends with the oocyst formation and excreted in feces. In chickens, seven species of Eimeria including E. tenella, E. necatrix, E. acervulina, E. maxima, E. brunetti, E. mitis, and E. praecox causes coccidiosis with variable levels of pathogenicity [3]. Identification and genetic characterization of different species of Eimeria genus are central to prevention, surveillance, and control of coccidiosis. This is particularly important with regard to the appearance of a widespread anticoccidial resistance of Eimeria species and the problems associated with drug residues. Identification of these parasites by observing oocyst morphology, prepatent period, site of infection or minimum sporulation time are labour-intensive, time consuming and can be very difficult and unreliable with mixed samples [4]. Due to difficulties in the morphological identification of some of chicken Eimeria spp., diagnostic laboratories are increasingly utilizing DNA-based technologies. Classical non-quantitative PCR method are already in use for molecular diagnosis of the seven Eimeria species that infect chickens [5, 6]. One such non-quantitative PCR methods is focused on amplifying sequence characterized amplified regions -SCARs [7]. So far, there is limited knowledge on the epidemiology of Eimeria in Bangladesh. The aim of this study was to detect different chicken Eimeria species from poultry litter through PCR approach that target unique single copy sequences derived from SCAR markers.

\section{Materials and Methods}

\section{Isolation and washing down of Eimeria oocysts from litter samples}

This research work was conducted with litter samples from broiler farms where coccidiosis is suspected based on bloody droppings. Total 18 litter samples were collected manually from different poultry farms located in different parts of Chittagong Metropolitan area in Southern Bangladesh. Fresh litters were collected by random sampling from 18 broiler chickens flocks with or without previous exposure to anticoccidial vaccines. Oocysts were excreted from the host via the feces. For this study the previously established protocol (Collected from Royal Veterinary College, London, UK) were used. The protocol describes the collection of fecal material, together with the purification of oocysts from it through soaking in water, mixing with a blender and separation 
of coarse materials by sieving. A combined centrifugation and salt floatation step was incorporated to separate the oocysts from fecal materials based upon a second criterion: specific gravity. The recovered oocysts were then washed out of salt and sporulated. After purification, the oocysts were preserved in $2.5 \%$ potassium dichromate at $28^{\circ}$. The potassium dichromate was removed before these oocysts were used for DNA extraction for subsequent use for PCR analyses.

\section{Parasitologic examination}

Using a calibrated ocular micrometer at 400x magnification, a modified saturated salt floatation technique was used to isolate oocysts for length measurements [8]. Fifty random oocysts from each sample were examined by measuring their length and width with light microscopy, armed with calibrate ocular lens as well as determination of the oocysts shape and index (Length/Width). Sporocysts' diameters were also determined for more accurate diagnosis [9].

\section{Genomic DNA preparation}

Purified oocysts were washed in PBS, disrupted using $0.5 \mathrm{~mm}$ glass beads and DNA was extracted from the lysate using the Qiagen Mini Stool DNA Kit (Qiagen, Germany) according to the manufacturer's instructions and stored at $-20^{\circ} \mathrm{C}$. Extracted DNA concentration was measured by Thermo scientific Nano drop 2000 spectrophotometer (USA)

\section{PCR amplification}

PCR targets were designed using the SCARdb database of species-specific SCARs described before [4]. The markers were designed in non-repetitive regions of each SCAR identified using Tandem repeats finder version 4.00 as a screen. The length of each PCR target was chosen to be between 100 and 150 bp. Primers and probes were designed using Primer3 software (Table 1).

Reactions were performed using GoTaq Green Mastermix (Promega, USA), 500uM forward and reverse primers and $5 \mathrm{ul}$ template DNA in a 20ul volume and run using a Applied Biosystem PCR thermal cycler (USA). For positive controls, Paracox ${ }^{\circledR}$ a commercial vaccine, which included all the eight pathogenic Eimeria species of chicken was used and water was used as negative control in PCR. The thermal cycling program consisted of initial denaturation at $95^{\circ} \mathrm{C}$ for 4 min followed by 40 cycles of denaturation at $94{ }^{\circ} \mathrm{C}$ for $30 \mathrm{~s}$, annealing at $58^{\circ} \mathrm{C}$ for $30 \mathrm{~s}$ and extension at $68^{\circ} \mathrm{C}$ for $2 \mathrm{~min}$. The final extension was set at $68{ }^{\circ} \mathrm{C}$ for $5 \mathrm{~min}$. To verify the results, $10 \mu 1$ of each PCR product was electrophoresed in a $1.5 \%$ agarose gel (Cat no. \# DV3123, Promega ${ }^{\circledR)}$ ), stained with SYBR Green Fuorescent Dye (Sigma, USA), and visualized on a UV transilluminator (Biometra, Germany). The PCR products were identified by size using a 100 base pair ladder (Cat. no\# RD002, RBC Bioscience, Taiwan).

Table 1: List of species specific primers used during this study[4]

\begin{tabular}{|c|c|l|c|}
\hline Species & Primer ID & \multicolumn{1}{|c|}{ Primer } & $\begin{array}{c}\text { Amplicon } \\
\text { size (bp) }\end{array}$ \\
\hline E. acervulina & Ac-AD18-953 & $\begin{array}{l}\text { ACE-F: GCAGTCCGATGAAAGGTATTTG } \\
\text { ACE-R: GAAGCGAAATGTTAGGCCATCT }\end{array}$ & 103 \\
\hline E. runetti & Br-J18-626 & $\begin{array}{l}\text { BRU-F: AGCGTGTAATCTGCTTTTGGAA } \\
\text { BRU-R: TGGTCGCAGACGTATATTAGGG }\end{array}$ & 118 \\
\hline E. maxima & EmMIC1 & $\begin{array}{l}\text { MAX-F: TCGTTGCATTCGACAGATTC } \\
\text { MAX-R: TAGCGACTGCTCAAGGGTTT }\end{array}$ & 138 \\
\hline E. mitis & Mt-A09-716 & $\begin{array}{l}\text { MIT-F: CAAGGGGATGCATGGAATATAA } \\
\text { MIT-R: CAAGACGAATGGAATCAATCTG }\end{array}$ & 115 \\
\hline E. necatrix & Nc-AD10-702 & $\begin{array}{l}\text { NEC-F: AACGCCGGTATGCCTCGTCG } \\
\text { NEC-R: GTACTGGTGCCAACGGAGA }\end{array}$ & 134 \\
\hline E. praecox & Pr-A09-1108 & $\begin{array}{l}\text { PRA-F: CACATCCAATGCGATATAGGG } \\
\text { PRA-R: ACAGAAAAACGCAAAGAGCAA }\end{array}$ & 117 \\
\hline E. tenella & Tn-E03-1161 & $\begin{array}{l}\text { TEN-F: TCGTCTTTGGCTGGCTATTC } \\
\text { TEN-R: CAGAGAGTCGCCGTCACAGT }\end{array}$ & 100 \\
\hline
\end{tabular}

\section{Microscopic examination and oocyst count}

The aim of the study was to use modern molecular biological tools to diagnose Eimeria species causing chicken coccidiosis in Bangladesh. Following standard protocol, the oocysts were collected from selected poultry farms with history of bloody diarrhea and moist litter. A modified saturated salt flotation technique was used to isolate oocysts for micrometry using a calibrated ocular micrometer at 400x magnification. Using a camera fitted with microscope, images of different oocysts were captured where three species of Eimeria including E. tenella, E. acervulina, E. maxima were ovserved clearly. Several other oocysts were not possible to identify based on size itself. Among three observed species E. tenella was predominant. 
The numbers of oocysts were counted using McMaster technique as found in different isolates The concentration of oocysts were different in each isolate which were from 1600-300,000.

\section{Results of PCR}

The amplified PCR products from different field isolates of oocyst materials were run in $1 \%$ agarose gel. Fig.1. indicates the corresponding amplified band in different isolates. During this study it was found that seven species namely E. tenella, E. necatrix, E. acervulina, E. maxima, E. brunetti, E. mitis, and E. praecox were successfully detected among 18 field samples. The frequency of different species was variable in different isolates where number of $E$. tenella was comperatively more $(\mathrm{n}=9)$, while occurrence of both $E$. necatrix and $E$. praecox was similar $(\mathrm{n}=8)$, E. brunette and $E$. mitis were fewer $(\mathrm{n}=5)$ and least recorded species were $E$. acervulina $(\mathrm{n}=3)$ and E. maxima $(\mathrm{n}=1)$.

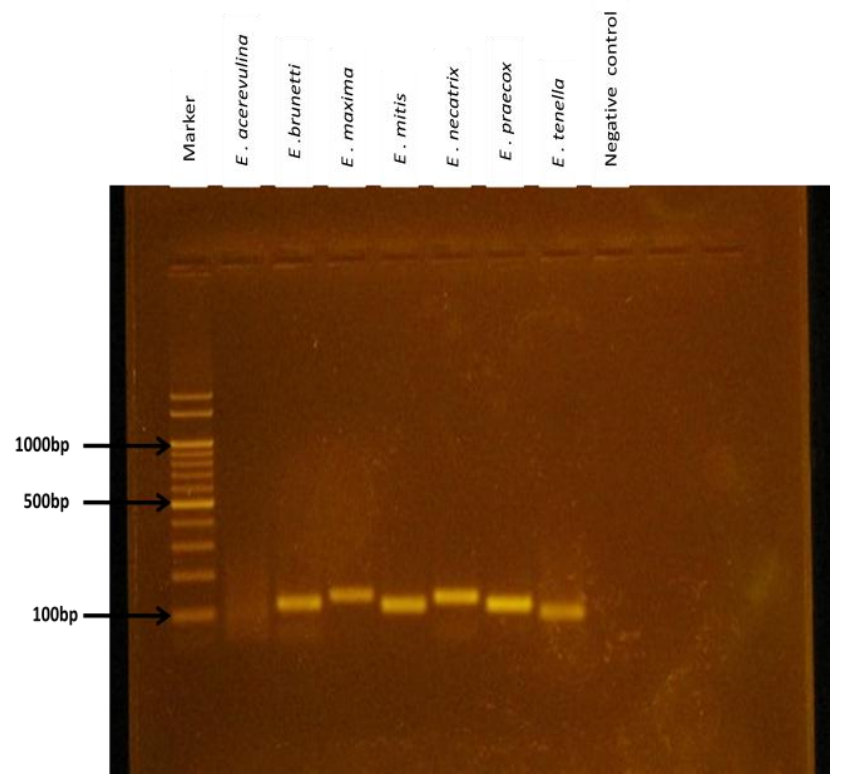

Fig 1 Agarose gel electrophoresis of Eimeria species-specific PCR products

\section{Discussion}

Coccidiosis is considered as one of the most economically important diseases of domestic poultry that is responsible for significant economic losses to the worldwide poultry industry [10]. It remains one of the major diseases problems of poultry industry spite of advances made in prevention and control through chemotherapy, management and nutrition [11]. E. tenella and E. necatrix are the most pathogenic species. Coccidiosis is caused by one or several of seven Eimeria species infecting chickens [10]. These species differ in their localization in the gut and in their ability to induce morbidity and mortality $[6,12]$. E. acervulina, E. maxima and E. mivati are common and slightly to moderately pathogenic; E. brunette is uncommon but pathogenic when it does occur. E. mitis, E. praecox and $E$. hagani are relatively non-pathogenic species [13, 14]. To our knowledge, E. mivati and E. hagani are not yet reported in Bangladesh.

Many factors may interfere in the success and effectiveness of diagnosis by PCR, especially in regards to the presence of contamination. According to Haug et al., [6] the DNA extraction process in stool samples is influenced by the formation of inhibitors of Taq DNA polymerase that affect the reaction. During this study, contaminated litter samples were collected from different poultry farms located in various regions of Chittagong in Bangladesh. The oocysts of Eimeria spp. were then purified using previously described protocols. While none of these protocols were $100 \%$ satisfactory for DNA based analysis, we have tried to optimize the DNA extraction from the experimental field samples during this study. Commercially available kit (Qiagen QIAamp DNA mini kit) was used for efficient collection of purified DNA without any inhibitors for PCR analysis. The quantity of extracted DNA was indicative of success in purified DNA which was later used as template.

A modified saturated salt floatation technique was used to isolate oocysts for micrometry using a calibrated ocular micrometer at 400x magnification. The protocol was validated by the 
coccidian research group of RVC (UK) and similar protocols have been followed by other workers. Once the oocysts were collected, classical tools like microscopy was followed. In absence of postmortem analysis and pathological data, it would be advantageous to measure the oocysts and identify the species. While the micrometry alone is not conclusive, molecular data were compared and validated to verify the oocysts from different species during this study. The concentration of oocysts were different in each isolate. Therefore the template concentration was measured and similar amount of DNA were used for optimized PCR analysis. The morphometric study of oocyst has confirmed three species of Eimeria including E. tenella, E. acervulina, E. maxima clearly and there also found some undescribed species. However, molecular study was successful in confirming the presence of all seven Eimeria species namely, E. acervulina, E. brunetti, E. maxima, E. mitis, E. necatrix, E. praecox, E. tenella. To our knowledge, this was the first attempt in the country where DNA based tools were used to successfully characterize the chicken Eimeria spp.

Species diagnosis is particularly important for planning an effective prevention and control program for coccidiosis [15]. Traditionally, diagnosis has been achieved by detecting Eimeria oocysts excreted in the feces of chickens by measuring oocyst and sporocyst dimensions or assessing the site and extent of the pathological lesions in the intestine of chickens [16]. In our present study we have followed the traditional microscopic method. Although the microscopic examinations can absolutely show the negative fecal samples, such traditional methods have generally had major limitations in the specific diagnosis of coccidiosis and identification of Eimeria species. These approaches are unreliable, particularly when multiple species of Eimeria simultaneously infect a single host and there is overlap in the size range and similarity in shape of oocysts and the sites of infection in the intestines are common [17]. For example, E. tenella reproduces in the caeca and adjacent intestine, and produces bloody lesions. E. maxima and E. necatrix both develop in the midsmall intestine, but should in theory be distinguishable based on the different sizes of their oocysts. Therefore the present study was focused on using DNA based tools which has high discriminatory power to characterize different species even when there are mixed infection with multiple species. The technique has high accuracy and therefore more reliable for confirmatory diagnosis.

The PCR approach enables the selective amplification of DNA from complex genomes. In brief, the principle of the technique is that the double-stranded genomic DNA template is denatured by heating, and the temperature is then decreased to allow one or more oligonucleotide primers to hybridize (anneal) to their complementary sequences on opposite strands of the template; the template-directed DNA synthesis (extension) then proceeds in both orientations from the primer sites by enzymatic catalysis with a thermostable DNA polymerase and results in the production of doublestranded products [18]. This synthesis is usually repeated 20-40 times in an automated thermal cycler. In each cycle, the template is replicated by a factor of two, such that, upon completion of the cycling, millions of copies of the DNA target are available for subsequent analyses. Many different PCR approaches are available such as nested PCR, multiplex PCR and real time PCR [19, 20]. During this study we have used direct PCR amplification of highly conserved region which was already proved highly specific by previous reports [4].

Several PCR based assays targeting different regions of the Eimeria genome have been described, such as the 5S rRNA, the small subunit rRNA [21, 22], the sporozoite antigen gene EASZ 240/160 [23] and ITS-1 [24, 5] and ITS-2 [25, 26, 27] genomic regions. Therefore the choice of gene is crucial for any successful PCR analysis. During this study the SCAR markers were used which is highly specific and discriminatory compared to other similar target regions. The SCARs are PCRbased markers that represent genomic DNA fragments at genetically defined loci that are identified by PCR amplification using sequence specific oligonucleotide primers [28, 29, 30]. As SCARs are primarily defined genetically, they can be used both as physical landmarks in the genome and as genetic markers [31]. The present study was able to successfully characterize seven species of Eimeria through SCAR marker based PCR approach. PCR targets were designed using the SCARdb database of species-specific SCARs [32].

\section{Conclusion}

To our knowledge the present study is the first of its type to use molecular tools to identify Eimeria spp from chickens in Bangladesh. The detection or specific diagnoses of coccidiosis in 
chickens by traditional technique are not sufficiently reliable and PCR based method of DNA sequence could resolve this problem. Although classical approach is less expensive and no highly technical instruments and facility will be required, the sensitivity of the test is compromised and their might be more false positive cases reported. The present study was able to verify that that modern molecular tools might replace the traditional approach provided all the technical facilities are available in any lab settings. The knowledge can be invaluable in developing species-specific single strain or multi-strain vaccine. The data also can be helpful to conduct molecular epidemiological investigation of coccidiosis in the whole country or in the region.

\section{Reference:}

[1]. Shirley M, Smith A, Tomley F.(2005): The Biology of Avian Eimeria with an emphasis on their Control by Vaccination. Adv. Parasitol, 60, 285-330.

[2]. Williams RB. (2005): Intercurrentcoccidiosis and necrotic enteritis of chickens: Rational, integrated disease management by maintenance of gut integrity. Avian Pathol, 34(3), 159-180.

[3]. McDougald LR, Reid WM. (1997): Coccidiosis. In: Diseases of Poultry. Ames Publishing, Iowa State University Press, IA, USA, 865883.

[4]. Vladmir V, Damer P, Blake, Poplstein M. (2010). Quantitative real-time PCR assays for detection and quantification of all seven Eimeria species that infect the chicken. Veterinary Parasitology, 174(3-4), 183-190.

[5]. Schnitzler BE, Thebo PL, Tomley FM, Uggla A, Shirley MW.(1999): PCR identification of chicken Eimeria : a simplified read-out. Avian Pathol, 28(1), 89-93.

[6]. Haug A, Thebo P, Mattsson JG. (2007): Asimplified protocol for molecular identification of Eimeria species in field samples. Vet. Parasitol, 146(1-2), 35-45.

[7]. Fernandez S, Pagotto AH, Furtado M, Katsuyama AM, MadeiraAMBN, Gruber A. (2003): A multiplex PCR assay for the simultaneous detection and discrimination of the seven Eimeria species that infect domestic fowl. Parasitology, 127(4), 317-325.

[8]. Long PL, Reid WM. (1982): A guide for the diagnosis of coccidiosis in chickens. The University of Georgia, College of Agriculture Experiment Stations, Research report, 404, 1-17.

[9]. Conwa DP, McKenzie ME. (2007): Poultry coccidiosis, Diagnostic and testing procedures. 3th ed. Blackwell publishing; $2007: 7-11$.

[10]. McDougald LR. (2003): Coccidiosis. In Saif et al. (eds) Diseases of Poultry, Iowa State Press, Iowa, pp. 1001-1010.

[11]. GraatEAM,Ploeger HW, Henken AMG. Reilingh DV, Noordhuizen JPT, Van Beek NG. (1996): Effects of Initial Litter Contamination Level with Eimeria acervulina in Population Dynamics and Production Characteristics in Broilers. Vet Parasitol., 65(3-4), 223-232.

[12]. Morris GM, Woods WG, Richards DG, Gasser RB. (2007). The application of a polymerase chain reaction (PCR)-based capillary electrophoretic technique provides detailed insights into Eimeria populations in intensive poultry establishments. Mol. Cell. Prob, 21(4), 288-294.

[13]. Soulsby EJL.(1982):Helminths, Arthropods and Protozoan's of Domesticated Animals, 7 th Edn. BailliereTindall, London, pp: 630.

[14]. Lillehoj HS, Trout JM.(1993): A Review of Recent Advances on Immunity and Vaccine Development. Avian Pathol., 22(1), 3-31.

[15]. Morris GM, Gasser RB. (2006): Biotechnological advances in the diagnosis of avian coccidiosis and the analysis of genetic variation in Eimeria . Biotechnology Advance, 24(6), 590-603.

[16]. Hamidinejat H, Shapouri MRS, Mayahi M, Borujeni MP. (2010): Characterization of Eimeria Species in Commercial Broilers by PCR Based on ITS1 Regions of rDNA. Iranian J Parasitol, 5(4): 48-54.

[17]. Long PL, Joyner LP.(1984): Problems in the identification of species of Eimeria . Journal of Parasitology, 31(4), 535-541.

[18]. Morris GM, Gasser RB. (2006): Biotechnological advances in the diagnosis of avian coccidiosis and the analysis of genetic variation in Eimeria . Biotechnology Advances, 24(6), 590-603.

[19]. Monis PT, Giglio S, Keegan AR, Thompson RCA. (2005): Emerging technologies for the detection and characterization of protozoan parasites. Trends Parasitol,21(7), 340-6.

[20]. Gasser RB. (2006). Molecular tools advances, opportunities and prospects. Vet Parasitol. 136(2), 69-89.

[21]. Stucki U, Braun R, Roditi I. (1993):Eimeria tenella: characterization of a 5S ribosomal RNA repeat unit and its use as a species specific probe. ExpParasitol, 76(1), 68-75.

[22]. Tsuji N, Kawazu S, Ohta M, Kamio T, Isobe T, Shimura K, Fujisaki K. (1997): Discrimination of eight chicken Eimeria species using the two-step polymerase chain reaction. J Parasitol, 83(5), 966-970.

[23]. Molloy JB, Eaves FW, Jeston PJ, Minchin CM, Stewart NP, Lew AE, Jorgensen WK. (1998): Detection of Eimeria acervulinausing the polymerase chain reaction. Avian Dis. 42(1), 119-123.

[24]. Schnitzler BE, Thebo P, Mattson JG, Tomley F, Shirley MW. (1998): Development of a diagnostic PCR assay for the detection and discrimination of four pathogenic Eimeria species of the chicken. Avian Pathol, 27(5), 490-497.

[25]. Gasser RB, Woods WG, Wood JM, Ashdown L, Richards G, Whithear KG. (2001): Automated, fluorescence-based approach for the specific diagnosis of chicken coccidiosis. Electrophoresis, 22(16), 3546-3550.

[26]. Lien YY, SheuSC, Liu HJ, Chen SC, Tsai MY, LuoSC,WuKC, Liu SS, Su HY. (2007): Cloning and nucleotide sequencing of the second internal transcribed spacer of ribosomal DNA for three species of Eimeria from chickens in Taiwan. The Veterinary Journal, 173(1), 184189.

[27]. Woods WG, WhithearKG, Richards DG, Anderson GR, Jorgensen WK, Gasser RB. (2000): Single-strand restriction fragment length polymorphism analysis of the second internal transcribed spacer (ribosomal DNA) for six species of Eimeria from chickens in Australia. International Journal of Parasitology, 30(9), 1019-1023.

[28]. Paran I, Michelmore RW. (1993). Development of reliable PCR-basedmarkers linked to downy mildew resistance genes in lettuce. TheorAppl Genet, 85, 985-999.

[29]. McDermott JM, Brandle U, Dutly F, Haemmerli UA, Keller S, Muller KE, Wolf MS. (1994): Genetic variation in powdery mildew of barley: development of RAPD, SCAR and VNTR markers. Phytopathology. 84, 1316-1321.

[30]. Agarwal M, Shrivastava N, Padh H. (2008): Advances in molecular marker techniques and their applications in plant sciences. Plant Cell Rep, 27(4), 617-631.

[31]. Chelkowski J, Stephen L. (2001): Molecular markers for leaf rust resistance genes in wheat. J Appl Genet, 42(2), 117-126.

[32]. Fernandez S, Katsuyama AM, Kashiwabara AY, Madeira AMBN, Durham AM, Gruber A.(2004): Characterization of SCAR markers of Eimeria spp. of domestic fowl and construction of a public relational database (The Eimeria SCARdb). FEMS Microbiol. Lett, 238(1), $183-188$. 